Intensivmed $2011 \cdot 48: 281-289$

DOI 10.1007/s00390-010-0238-6

Eingegangen: 21. Januar 2011

Angenommen: 1. März 2011

Online publiziert: 1. Mai 2011

(c) Springer-Verlag 2011
G.-C. Funk

Intensivstation, Erste Interne Lungenabteilung, Otto-Wagner-Spital, Wien

\section{Differenzialdiagnose und Management von Weaningproblemen}

Schema durchgeführt werden (• Abb. 1, [10]). Wenn Ursache und Ausmaß der respiratorischen Insuffizienz gebessert sind, sollte die Entwöhnbarkeit systematisch evaluiert werden. Ob die Durchführung eines Spontanatemversuchs sinnvoll ist, wird anhand bestimmter „Ready-towean"-Kriterien beurteilt (• Tab. 1). Die einzelnen Tests zur Objektivierung dieser Kriterien sind als Entscheidungshilfen zu verstehen und müssen nicht alle routinemäßig überprüft werden.
Wenn die „Ready-to-wean“-Kriterien erfüllt werden, erfolgt ein kurzer Spontanatemversuch als Screeningtest auf Entwöhnbarkeit: Der Spontanatemversuch wird klassischerweise unter $\mathrm{O}_{2}$-Insufflation über ein T-Stück über 2 Minuten durchgeführt. Alternativ wird häufig CPAP ["continuous positive airway pressure" mit einem positiven endexspiratorischen Druck (PEEP) von 5 mbar)] mit oder ohne automatischer Tubuskompensation oder auch mit einer geringen insten mit einer liegenden Trachealkanüle sind als entwöhnt zu betrachten, wenn sie die genannten Kriterien erfüllen.

Die Entwöhnung ist ein kritischer Teil der Beatmungstherapie und nimmt bis zur Hälfte der gesamten Beatmungszeit ein [1]. Eine zeitgerechte Entwöhnung ist für den Patienten entscheidend, da eine Re-Intubation nach verfrühter Extubation mit einem erhöhten Pneumonierisiko und einer erhöhten Sterbewahrscheinlichkeit einhergeht. [2, 3, 4]. Auf der anderen Seite erhöht jedoch auch eine unnötig verzögerte Entwöhnung die Morbidität und Mortalität $[5,6,7]$.

\section{Entwöhnung von der Beatmung}

\section{Allgemeines Vorgehen und Einteilung}

Ärzte neigen dazu, die Entwöhnbarkeit von Patienten $\mathrm{zu}$ unterschätzen und $\mathrm{zu}$ spät zu erkennen $[8,9]$. Daher sollte die Entwöhnung schon sehr früh im Verlauf einer Beatmung in Betracht gezogen und dann strukturiert nach einem klaren
Abb. 1 Schrittweise Entwöhnung von der Beatmung. Die Berücksichtigung der Vortest-Wahrscheinlichkeit („Ready-to-wean"-Kriterien) und die sequenzielle Verwendung eines Screeningtests und eines Bestätigungstests erhöhen die Wahrscheinlichkeit einer frühen und erfolgreichen Entwöhnung mit niedriger Re-Intubationsrate. $R S B I^{\prime \prime}$ Rapid shallow breathing index": Atemfrequenz (Atemzüge / $\mathrm{min}$ ) / Tidalvolumen (I)

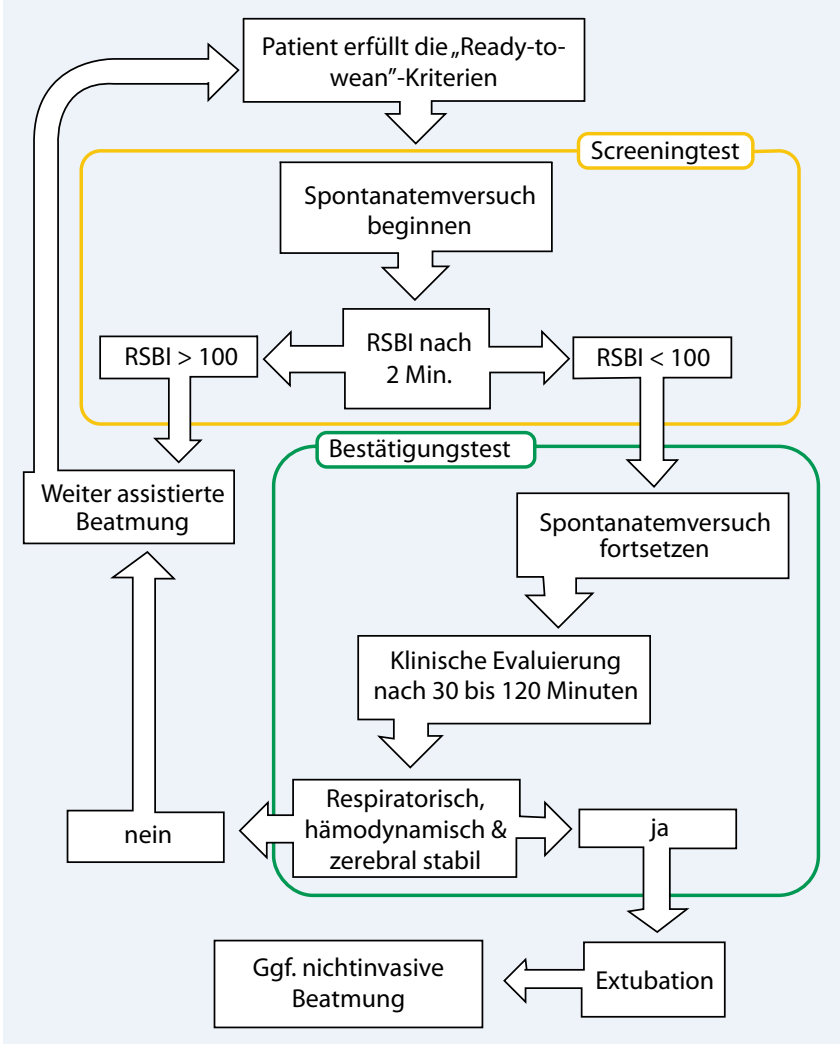




\begin{tabular}{|c|c|}
\hline \multicolumn{2}{|l|}{ Kriterien } \\
\hline Prinzipielle Voraussetzung & Ursache der respiratorischen Insuffizienz (z. B. Pneumonie) gebessert \\
\hline \multicolumn{2}{|c|}{ Subjektive und objektive Kriterien } \\
\hline Stabile Hämodynamik & $\begin{array}{l}\text { Herzfrequenz }<140 / \mathrm{min} \text {, systolischer Blutdruck } 90-160 \mathrm{mmHg} \text {, keine } \\
\text { oder minimale Vasopressoren und/oder i.v.-Inotropika } \\
\text { Keine Myokardischämie in den letzten } 24 \text { Stunden } \\
\text { Keine wirksamen Arrhythmien }\end{array}$ \\
\hline Adäquater Gasaustausch & $\begin{array}{l}\mathrm{S}_{\mathrm{a}} \mathrm{O}_{2} \geq 88 \% \text { bei } \mathrm{F}_{1} \mathrm{O}_{2} \leq 50 \% \text { bei PEEP } \leq 8 \mathrm{mbar} \\
\mathrm{p}_{\mathrm{a}} \mathrm{CO}_{2} \leq 50 \mathrm{mmHg}\end{array}$ \\
\hline $\begin{array}{l}\text { Adäquater Atemantrieb und } \\
\text { Atemmechanik }\end{array}$ & $\begin{array}{l}\text { Spontane Atmung vorhanden } \\
\text { Spontane Atemfrequenz }<35 / \mathrm{min} \\
\text { Tidalvolumen } \geq 5 \mathrm{ml} / \mathrm{kg} \text { ideales } \mathrm{KG} \\
\text { Ausreichender Hustenstoß (>35 l/min) } \\
\text { Keine exzessiven respiratorischen Sekrete (endotracheales Absaugen } \\
\text { nicht häufiger als maximal zweistündlich erforderlich) } \\
\text { MIP } \geq 30 \mathrm{CmH}_{2} \mathrm{O}\end{array}$ \\
\hline Adäquate zerebrale Funktion & $\begin{array}{l}\text { Patient kontaktierbar (und kooperativ) } \\
\text { Keine oder geringe Sedierung } \\
\text { Kein Hinweis für erhöhten Hirndruck }\end{array}$ \\
\hline \multicolumn{2}{|c|}{$\begin{array}{l}\text { Die angeführten Kriterien sind als Entscheidungshilfen zu verstehen und müssen nicht alle routinemäßig } \\
\text { überprüft werden. MIP maximale inspiratorische Atemwegsdrucknegativierung; PEEP positiver endexspiratori- } \\
\text { scher Druck. }\end{array}$} \\
\hline
\end{tabular}

Tab. 2 Kriterien eines fehlgeschlagenen (negativen) Spontanatemversuchs. (Mod. nach [10, 20])

\begin{tabular}{|c|c|}
\hline \multicolumn{2}{|l|}{ Befunde } \\
\hline $\begin{array}{l}\text { Klinische Be- } \\
\text { urteilung }\end{array}$ & $\begin{array}{l}\text { Agitation und Panik } \\
\text { Neu aufgetretene Somnolenz oder Koma } \\
\text { Verstärktes Schwitzen }\end{array}$ \\
\hline \multicolumn{2}{|c|}{ Weitere Messwerte und Kriterien } \\
\hline Gasaustausch & $\begin{array}{l}\mathrm{S}_{\mathrm{a}} \mathrm{O}_{2}<88 \% \text { bei } \mathrm{F}_{1} \mathrm{O}_{2} \leq 50 \% \text { über } 5 \mathrm{~min} \\
\text { Anstieg des } \mathrm{p}_{\mathrm{a}} \mathrm{CO}_{2}>10 \mathrm{mmHg}\end{array}$ \\
\hline $\begin{array}{l}\text { Atemmecha- } \\
\text { nik und Atem- } \\
\text { pumpe }\end{array}$ & $\begin{array}{l}\text { Anstieg des } \mathrm{RSBI} \geq 20 \% \text { im Vergleich zum Beginn des SBTs } \\
\text { Atemfrequenz }<8 / \text { min über } 5 \text { min } \\
\text { Einsatz der inspiratorischen und/oder exspiratorischen Atemhilfsmuskeln } \\
\text { Abdominales Paradoxon (paradoxe Atmung) } \\
\text { Massive Dyspnoe }\end{array}$ \\
\hline $\begin{array}{l}\text { Herz und Hä- } \\
\text { modynamik }\end{array}$ & $\begin{array}{l}\text { Herzfrequenz }>140 \text { oder }<60 / \text { min oder Anstieg } \geq 20 \% \text { im Vergleich zu Beginn des } S B T s \\
\text { Systolischer Blutdruck }>180 \mathrm{mmHg} \text { oder }<90 \mathrm{mmHg} \text { oder Anstieg oder Abfall } \geq 20 \% \\
\text { im Vergleich zu Beginn des SBTs } \\
\text { Neu aufgetretene Arrhythmie } \\
\text { Neu aufgetretene Angina pectoris }\end{array}$ \\
\hline RSBI"rapid shallo & w breathing index": Verhältnis Atemfrequenz (Atemzüge/min) / Tidalvolumen (I); SBT Spontan- \\
\hline
\end{tabular}

piratorischen Druckunterstützung von 5-8 mbar („pressure support ventilation“) verwendet [11]. Das Verhältnis Atemfrequenz (Atemzüge pro Minute) / Tidalvolumen $(\mathrm{l})=$ „rapid shallow breathing index“ (RSBI) am Ende dieser kurzen Spontanatemphase ist ein guter Prädiktor der Entwöhnbarkeit und entscheidet über das weitere Vorgehen [12].

- Bei einem RSBI >100 besteht eine äu-

Berst niedrige Wahrscheinlichkeit von

Entwöhnbarkeit und der Patient wird

weiter assistiert beatmet.
- Wenn der Spontanatemversuch über 30 Minuten positiv verläuft, kann der Patient mit hoher Wahrscheinlichkeit erfolgreich extubiert werden.

Wenn hingegen Zeichen der respiratorischen, hämodynamischen oder zerebralen Instabilität auftreten, so wird der Spontanatemversuch mit einem negativen Ergebnis abgebrochen, und der $\mathrm{Pa}$ tient wird weiter assistiert beatmet. Bis zum nächsten Spontanatemversuch (üblicherweise am Folgetag) sollte die Atempumpe ausreichend entlastet werden, jedoch nicht vollständig ruhen [13]. Sedierung und kontrollierte Beatmung sind in diesem Zusammenhang vermutlich kontraproduktiv. Wenn bereits einer oder mehrere vorangegangene Spontanatemversuche negativ ausgefallen sind, so muss ein weiterer Spontanatemversuch über 2 Stunden (und nicht nur 30 Minuten) positiv verlaufen, bevor der Patient extubiert werden kann.

Anhand der Anzahl der für eine erfolgreiche Entwöhnung erforderlichen Spontanatemversuche und anhand der Anzahl der dafür benötigten Tage wird die Entwöhnung in einfach, erschwert und prolongiert eingeteilt (• Abb. 2, [10]). Die Mehrzahl der Patienten hat eine einfache oder erschwerte Entwöhnung und eine niedrige Mortalität [14].

Patienten mit chronischen Lungenkrankheiten, die während des Spontanatemversuchs eine Hyperkapnie mit $\mathrm{p}_{\mathrm{a}}$ $\mathrm{CO}_{2}>45 \mathrm{mmHg}$ entwickeln, profitieren nachweislich von einer prophylaktischen nichtinvasiven Beatmung unmittelbar nach der Extubation $[15,16]$. Ähnliches gilt für Patienten mit Herzinsuffizienz [17]. Voraussetzungen für eine erfolgreiche nichtinvasive Beatmung sind ein hämodynamisch stabiler und kooperativer Patient sowie ausreichende Expertise des Personals mit der nichtinvasiven Beatmung. Wenn Patienten nach der Extubation weiterhin eine nichtinvasive Atemhilfe benötigen spricht man von „Weaning-inprogress“ [10]. Wenn die Atmung innerhalb von 48 Stunden nach Extubation stabil geblieben ist, spricht man von einer erfolgreichen Extubation und einer erfolgreichen Entwöhnung [9, 18].

Nach der Extubation muss der $\mathrm{Pa}$ tient weiter hinsichtlich Zeichen der res- 
piratorischen Erschöpfung monitiert werden (• Tab. 2). Eine manifeste Erschöpfung der Atempumpe ist in jedem Fall zu vermeiden, da deren Erholung Stunden bis Tage benötigt. Vielmehr sollte bei den ersten Anzeichen der respiratorischen Erschöpfung eine neuerliche Atemhilfe etabliert werden. Dies impliziert jedoch nicht automatisch eine Re-Intubation, sondern sollte primär mit nichtinvasiver Beatmung gewährleistet werden.

\section{Prolongierte Entwöhnung}

\section{Häufigkeit und Prognose}

Bei etwa $15 \%$ aller entwöhnungsfähigen Patienten, kommt es zu einer prolongierten Respiratorentwöhnung [10, 14]. Diese Patienten haben eine gesteigerte kurzund mittelfristige Mortalität [14] und benötigen bis zu der Hälfte der finanziellen Ressourcen einer Intensivstation [21]. Nach einem halben Jahr sind etwa zwei Drittel dieser Patienten erfolgreich entwöhnt und nach einem Jahr sind zwei Drittel noch am Leben [22]. Patienten mit einer zugrundeliegenden chronisch-obstruktiven Lungenkrankheit (COPD) haben eine schlechtere Prognose als Patienten, die postoperativ oder nach einem akuten Lungenversagen (ARDS) prolongiert entwöhnt wurden [23, 24]. Bei drei Viertel der im Rahmen von prolongierter Beatmung verstorbenen Patienten wurde eine Therapiebegrenzung oder ein Therapieabbruch durchgeführt [22].

\section{Ursachen}

Bereits bei jenen Patienten, die eine erschwerte Entwöhnbarkeit zeigen, spätestens aber bei Patienten mit prolongierter Entwöhnung, ist eine systematische Suche nach der oder den Ursachen erforderlich. Es empfiehlt sich eine systematische Beurteilung der einzelnen physiologischen Systeme unter Berücksichtigung der Vorerkrankungen, der aktuellen klinischen Befunde und zusätzlicher bildgebender und funktioneller Diagnostik (• Tab. 3).

\section{Respiratorische Limitation}

Als Folge der pathologischen Atemmechanik (erhöhter Flusswiderstand und/ oder erhöhter elastischer Widerstand der

Intensivmed 2011 - 48:281-289 DOI 10.1007/s00390-010-0238-6

(C) Springer-Verlag 2011

G.-C. Funk

Differenzialdiagnose und Management von Weaningproblemen

Zusammenfassung

Bei einem von sechs beatmeten Patienten verläuft die Entwöhnung vom Respirator (Weaning) prolongiert, was mit einer deutlich erhöhten Morbidität und Mortalität einhergeht. Bereits nach dem ersten gescheiterten Spontanatemversuch sollte nach dem oder den die Entwöhnung verhindernden Organsystem(en) gefahndet werden. Die konkrete Ursache von schwierigem Weaning kann am besten am Höhepunkt eines gescheiterten Spontanatemversuchs anhand der klinischen Untersuchung, der arteriellen und venösen Blutgase, der Echokardiographie und anderer Hilfsmittel festgestellt werden. Pathologische Atemmechanik, schwache bzw. überlastete Atemmuskulatur, Herzinsuffizienz und Myokardischämie sowie psychi- sche Probleme können an bestimmten Befundkonstellationen erkannt werden. Reduktion der Atemarbeit, Training der Atemmuskulatur und Senkung von Vor- und Nachlast sind die entscheidenden Schritte zur Überwindung einer respiratorisch bzw. kardial limitierten Entwöhnbarkeit. Erfolgreiche Strategien zur Vermeidung von Weaningproblemen sind der sparsame Umgang mit Sedierung, der frühe Beginn mit einer strukturierten Entwöhnung sowie die frühe Rehabilitation schwacher Patienten.

\section{Schlüsselwörter}

Entwöhnung · Beatmung - Tracheotomie · Intensivstation

\section{Diagnosis and management of difficult weaning from mechanical ventilation}

Abstract

Of 6 ventilated ICU patients, 1 patient will experience prolonged weaning from mechanical ventilation. This is associated with a substantially increased morbidity and mortality. After one unsuccessful spontaneous breathing trial, the organ system preventing successful weaning should be identified. The mechanisms limiting weaning should be assessed toward the end of a failing spontaneous breathing trial using physical examination, arterial and venous blood gases, echocardiography, and other tools. Disturbed mechanics of the respiratory system, weakness of the ventilatory muscles, and weaning-induced cardiac dysfunction including myocar- dial ischemia are common causes of difficult or prolonged weaning. The respective therapeutic strategies are reduction of the work of breathing, ventilatory muscle training, and reducing cardiac preload as well as afterload. Weaning problems are preventable by a structured weaning strategy, including early spontaneous awakening and breathing trials as well as early rehabilitation of patients with ICU-acquired weakness.

\section{Keywords}

Ventilator weaning $\cdot$ Ventilation, mechanical . Tracheotomy · Intensive care units 


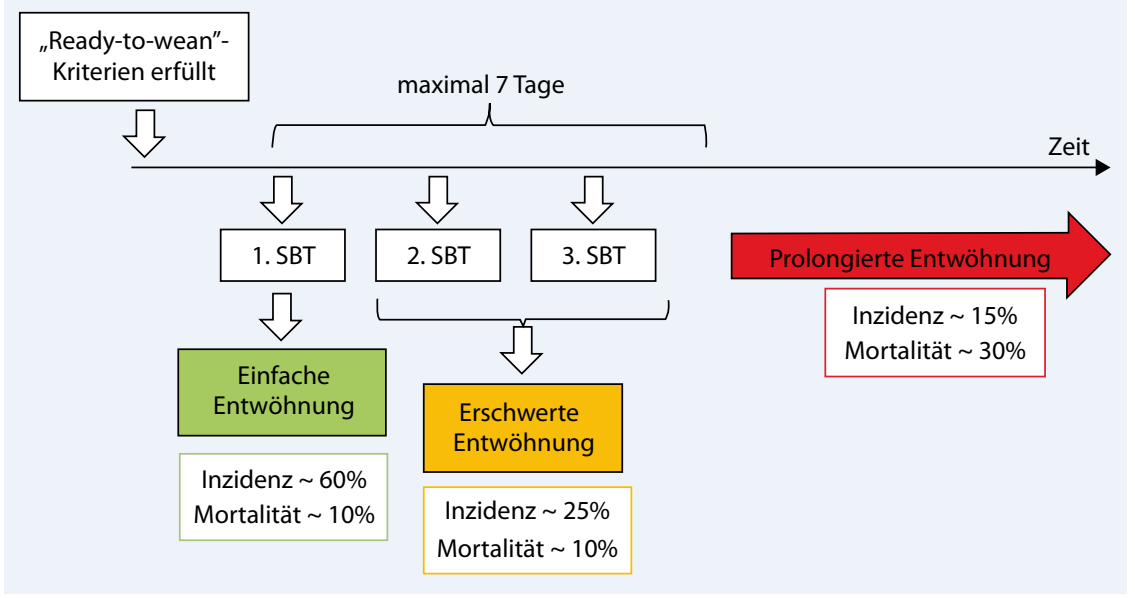

Abb. $2 \Delta$ Kategorisierung der Entwöhnung vom Respirator. SBT "spontaneous breathing trial", Spontanatemversuch. Einfache Entwöhnung: Erfolgreiche Extubation nach dem 1. SBT. Erschwerte Entwöhnung: 1. SBT verläuft negativ, aber erfolgreiche Extubation innerhalb von 7 Tagen beim 2. oder 3. SBT. Prolongierte Entwöhnung: Mehr als 3 SBTs verlaufen negativ oder die Entwöhnung benötigt insgesamt mehr als 7 Tage

Lunge bzw. des Thorax) bei verschiedenen akuten und chronischen Lungenkrankheiten ist die durch die Atempumpe (reguläre und akzessorische Atemmuskulatur) aufzubringende Last deutlich erhöht. Gleichzeitig ist oftmals die Kapazität der Atempumpe durch verschiedene Formen von Myopathie eingeschränkt. Es resultiert eine insuffiziente Atempumpleistung, was sich klinisch mit Tachypnoe, Dyspnoe, Einsatz der Atemhilfsmuskeln und mit einer Hechelatmung (,,rapid shallow breathing“) bemerkbar macht.

Die maximale Kraft der Atempumpe kann brauchbar abgeschätzt werden, indem der Druck am Atemweg während forcierter Inspiration gegen den verschlossenen Atemweg (Müller-Manöver) gemessen wird. Für eine detaillierte Vermessung der Belastung der Atempumpe ist ein Ösophagus- und/oder Magenballonkatheter erforderlich.

\section{() Die Kräftigung der Atemmuskulatur beschleunigt die Entwöhnung}

Die therapeutische Strategie bei insuffizienter Atempumpe umfasst einerseits die Reduktion der atemmechanischen Last (Bronchospasmolyse, Sekretabsaugung, Pleuraergusspunktion u. a.) und - sofern die Kraft der Atempumpe reduziert ist - ein Atemmuskeltraining [25, 26]. Eine Steigerung der Kraft der Atemmuskulatur unter der Rehabilitation geht mit einer er- folgreichen Entwöhnung einher [27]. Die Atempumpe kann mittels intermittierender kurzer Spontanatemphasen und/oder mit Atmung gegen einen dosierten Widerstand trainiert werden [26]. Das optimale Trainingsschema für diese Patienten ist bislang nicht bekannt. Zwischen den Trainingsphasen ist in jedem Fall eine ausreichende Entlastung der Atempumpe mittels assistierter Beatmung erforderlich.

Sekretretention bei Hustenschwäche ist ein weiterer häufiger Grund für verzögerte Respiratorentwöhnung. Ein Hustenspitzenstoß $<35 \mathrm{l} / \mathrm{min}$ ist mit prolongierter Entwöhnung assoziiert [28]. Entsprechende atemphysiotherapeutische Techniken zur Sekretmobilisierung und Sekretelimination sollen in solchen Fällen früh eingesetzt werden.

Beatmungsform und -muster. Während prolongierter Respiratorentwöhnung wird zwischen den Spontanatemphasen in der Mehrzahl der Fälle mit „pressure support ventilation“ (oder „assisted spontaneous breathing“, ASB) beatmet. Hier ist auf eine adäquate inspiratorische Druckunterstützung und die Vermeidung von PatientenRespirator-Dyssynchronien (u. a. Triggerversagen, Autotriggering, verfrühtes oder verzögertes Cycling) zu achten. Bei starker Dyssynchronie stehen als alternative Beatmungsformen ,proportional assist ventilation“ (besonders bei Cycling-Dyssynchronien) und NAVA („neurally-adjusted ventilatory assist“) zur Verfügung. Bei „pressure support“ hat sich ein Flowtrigger von $2-5 \mathrm{l} / \mathrm{min}$ und ein inspiratorischer Flow von etwa $601 / \mathrm{min}$ bewährt.

Ein zu geringer inspiratorischer Flow erhöht die Atemarbeit des Patienten, ein zu hoher Flow kann zu Tachypnoe mit Überblähung führen. Patienten mit COPD oder Asthma mit Airtrapping und intrinsischem PEEP (PEEPi) sollten mit einem externen PEEP von $80 \%$ des PEEPi beatmet werden. Bei spontanatmenden oder assistiert beatmeten Patienten kann der PEEPi nur mittels Ösophagusballonkatheter gemessen werden. Alternativ kann der optimale externe PEEP eruiert werden, indem der externe PEEP schrittweise gesteigert wird, bis die Anzahl der Einatembemühungen mit Triggerversagen minimiert ist.

\section{Kardiale Limitationen}

Bei einer Vielzahl von älteren Patienten besteht schon vor der kritischen Krankheit eine koronare Herzkrankheit und/ oder andere myokardiale oder valvuläre Probleme. Bei Umstellung auf Spontanatmung muss es obligatorisch zu einem Anstieg des Herzzeitvolumens und somit des myokardialen $\mathrm{O}_{2}$-Verbrauchs kommen, um den gesteigerten metabolischen Bedürfnissen der Atemmuskulatur gerecht zu werden. Gleichzeitig führt jedoch die Absenkung des intrathorakalen Drucks während der Spontanatmung zu einer Erhöhung der linksventrikulären Vor- und Nachlast, sowie zur Erhöhung der rechtsventrikulären Vorlast. Wenngleich die rechtsventrikuläre Nachlast bei Spontanatmung normalerweise sinkt, kann sie bei COPD und Asthma wegen der dynamischen Überblähung zunehmen. Patienten mit COPD zeigen während den Spontanatemversuchen in der Entwöhnung häufig neben dem pulmonalen Grundproblem eine zusätzliche kardiale Dysfunktion, welche auf die infolge der stark negativen Intrathorakaldrücke besonders starke Erhöhung der myokardialen Arbeitslast zurückzuführen ist [29].

\footnotetext{
- Als Folge der insgesamt gesteigerten kardialen Last kann während der Entwöhnung eine latente Herzinsuffizienz manifest werden.
} 
Tab. 3 Ursachen, Mechanismen, Diagnose und Management der prolongierten Respiratorentwöhnung

\begin{tabular}{|c|c|}
\hline Ursachen und Mechanismen & Klinische Hinweise und Diagnose \\
\hline \multicolumn{2}{|c|}{ Besteht ein Missverhältnis zwischen Last und Kapazität der Atempumpe? } \\
\hline & $\begin{array}{l}\text { Während des scheiternden SBTs: } \\
\text { - RSBI steigt an, deutlich > } 100 \\
\text { - Klinisch: Einsatz der inspiratorischen Atemhilfsmuskeln (z. B. } \\
\text { M. sternocleidomastoideus), Hoover-Zeichen, paradoxe At- } \\
\text { mung, inspiratorische Einziehung der Zwischenrippenräume } \\
\text { - Akuter Anstieg des } \mathrm{p}_{\mathrm{a}} \mathrm{CO}_{2} \text { mit Azidämie } \\
\text { - } \mathrm{S}_{\mathrm{cv}} \mathrm{O}_{2} \text { in Ruhe normal, sinkt am Ende des gescheiterten SBTs } \\
\text { weiter ab } \\
\text { - Ggf. Oxygenierungsstörung }\end{array}$ \\
\hline
\end{tabular}

Besteht eine erhöhte atemmechanische Last?

Erhöhte pulmonale Resistance

- COPD, Asthma

- Sekretretention

- Kardiogenes Lungenödem (Bronchialwandödem)

- Endobronchialer Tumor

Airtrapping mit PEEP

- COPD und Asthma

- Spontanatmung: Lippenbremse

Erniedrigte pulmonale Compliance

- Adipositas

- Kardiogenes Lungenödem

- ARDS, Pneumonie, Infiltrate, interstitielle Fibrose

- Pleuraerkrankungen (Erguss, Schwarte)

Erniedrigte extrapulmonale Compliance und/oder erhöhter

Pleuradruck

- Adipositas

- Intraabdominale Hypertension

- Thoraxwanddeformation (Kyphoskoliose, Thoraxplastik)

- Hyperhydratation, Ödeme

Besteht eine reduzierte Kraft der Atemmuskelpumpe?
Hinweise für COPD:

- Radiologisch Emphysemzeichen

- Auskultatorisch obstruktive Nebengeräusche

- Einsatz der exspiratorischen Atemhilfsmuskeln, z. B. M. transversus abdominis

- Exspiratorische Flowlimitation bei vorangegangener kontrollierter Beatmung

- „Missed-efforts" in der Flowkurve (d. h. nicht getriggerte Einatembemühungen wegen erhöhtem intrinsischem PEEP)

Thoraxsonographie

Messung der ösophagealen, intragastralen und/oder vesikalen Drücke
Spezifisches Management

Ursachen erhöhter Last finden und behandeln (s. unten) Etwaige Schwäche der Atemmuskulatur objektivieren und behandeln (s. unten)

Behandlung entsprechend dem spezifischen Problem
- Muskuläre Schwäche bei kritischer Krankheit

(„critical-illness acquired weakness ${ }^{\prime \prime}$ )

- Beatmungsassoziierte Zwerchfelldysfunktion

- Sekretretention bei Hustenschwäche
Risikofaktoren: Lange Beatmungsdauer, aggressive Beatmung, lange, tiefe Sedierung, Therapie mit Glukokortikoiden, Relaxierung

Objektivierung der Messung des MIP: Eine klinisch relevante Einschränkung der Atemmuskelkraft kann bei normalem MIP $>70 \mathrm{~cm} \mathrm{H}_{2} \mathrm{O}$ ausgeschlossen werden

Maximaler Hustenstoß $<35 \mathrm{I} / \mathrm{min}$
Training der Atemmuskulatur (z. B. intermittierende Spontanatemphasen ohne Respiratorunterstützung) und der Extremitätenmuskulatur

Atemphysiotherapie, bronchoskopische Sekretabsaugung, Tracheotomie

\section{Gibt es eine präexistente oder durch das Weaning induzierte kardiale Dysfunktion?}

- Koronare Herzkrankheit

- Herzinsuffizienz

- Vitien (v. a. Aortenstenose)

- Aggravierung einer pulmonalen Hypertension
Während eines scheiternden SBTs:

- Angina pectoris

- EKG-Streckenveränderungen

- $\mathrm{S}_{\mathrm{cv}} \mathrm{O}_{2}$ ggf. schon in Ruhe erniedrigt, sinkt am Ende des gescheiterten SBTs deutlich weiter ab

- Laktat steigt an (>2-3 mmol/l)

- Oxygenierungsstörung, ggf. Hyperkapnie

- Zeichen der dekompensierten Atempumpe sekundär möglich

- Radiologisch Lungenödem

- Anstieg von $E / E^{\prime}>8,5$ im Gewebedoppler

- BNP deutlich erhöht

- Bei scheiterndem SBT Anstieg des BNP > 20\% im Vergleich zu unmittelbar vor Beginn des SBTs

- Anstieg Gesamtprotein im Plasma >9\% am Ende des SBTs im

Vergleich zu unmittelbar vor Beginn des SBTs

- Ggf. Anstieg des ZVD

- PAP im Echo schätzen, ggf. invasiv messen
- Koronarischämie: Therapie der KHK (ggf. Revaskularisierung)

- Herzinsuffizienz: Vorlast senken durch Entwässerung und ggf. Nitroglycerininfusion während des SBTs

- Reduzierte Kontraktilität: Inotropika (z. B. Levosimendan)

- Vitien behandeln

- Ggf. Druck im kleinen Kreislauf senken 


\begin{tabular}{|c|c|c|}
\hline Ursachen und Mechanismen & Klinische Hinweise und Diagnose & Spezifisches Management \\
\hline \multicolumn{3}{|c|}{ Besteht eine neuropsychiatrische Limitation? } \\
\hline $\begin{array}{l}\text { - Delir } \\
\text { - Panikattacken }\end{array}$ & $\begin{array}{l}\text { - Anamnese, sichtbare Panik } \\
\text { - Hyperventilation mit Hypokapnie: } \mathrm{p}_{\mathrm{a}} \mathrm{CO}_{2}-\mathrm{Absinken} \\
\text { - } \mathrm{S}_{\mathrm{cv}} \mathrm{O}_{2} \text { normal unter SBT } \\
\text { - Lunge und Herz: morphologisch und funktionell keine Erklä- } \\
\text { rung für das Weaningversagen }\end{array}$ & $\begin{array}{l}\text { Delir behandeln } \\
\text { Beruhigen, Biofeedback } \\
\text { Neuroleptika (z. B. Risperi- } \\
\text { don), eher keine Benzodia- } \\
\text { zepine }\end{array}$ \\
\hline \multicolumn{3}{|l|}{ Besteht ein reduzierter Atemantrieb? } \\
\hline $\begin{array}{l}\text { - Opioide } \\
\text { - Restsedierung } \\
\text { - Metabolische Alkalose mit Alkalämie } \\
\text { - Hirnstammläsion }\end{array}$ & $\begin{array}{l}\text { - Fehlender oder lediglich geringer Anstieg von Atemfrequenz } \\
\text { und Atemzugvolumen während des SBTs } \\
-\mathrm{P}_{0,1}<1 \mathrm{mbar}\end{array}$ & Alkalose: Azetazolamid \\
\hline \multicolumn{3}{|c|}{ Bestehen metabolische oder endokrine Störungen? } \\
\hline \multicolumn{3}{|c|}{$\begin{array}{l}\text { - Elektrolytstörungen, v. a. Dysnatriämien, Hypophos- } \\
\text { phatämie } \\
\text { - Hypothyreose, Hypoaldosteronismus }\end{array}$} \\
\hline \multicolumn{3}{|c|}{ Besteht ein Ernährungsproblem? } \\
\hline $\begin{array}{l}\text { - Vorbestehende Malnutrition } \\
\text { - Ungenügende Kalorienzufuhr }\end{array}$ & $\begin{array}{l}\text { Kachexie, Hypoalbuminämie, Cholinesterase nachhaltig } \\
\text { reduziert }\end{array}$ & \\
\hline \multicolumn{3}{|c|}{$\begin{array}{l}\text { RSBI „rapid shallow breathing index“: Atemfrequenz (Atemzüge / min) / Tidalvolumen (I); } \mathrm{S}_{\mathrm{cV}} \mathrm{O}_{2} \text { Sauerstoffsättigung in der oberen Hohlvene; BNP „brain natriuretic peptide“; } \\
\text { E frühe diastolische Geschwindigkeit des orthograden Mitralklappenflusses (PW Doppler); E' frühe diastolische Geschwindigkeit im Mitralklappenring (Gewebedoppler); } \\
\text { MIP maximaler inspiratorischer Druck (Druck am Atemweg, während forcierter Inspiration gegen den verschlossenen Atemweg gemessen); } \mathrm{P}_{0,1} \text { Atemwegsokklusionsdruck } \\
\text { in } 100 \text { ms; PAP Pulmonalisdruck; PEEPi intrinsischer PEEP = auto-PEEP; SBT Spontanatemversuch; ZVD zentraler Venendruck. }\end{array}$} \\
\hline
\end{tabular}

Dies kann sich als Lungenödem, aber auch als Blutdruckabfall und/oder Anstieg des Laktats äußern. Eine Abnahme der gemischt-venösen oder zentral-venösen Sättigung während eines Spontanatemversuchs, sowie der Anstieg des BNP („brain natriuretic peptide") sind Hinweise für ein drohendes kardiales Versagen [30, 31, 32]. Steigende linksventrikuläre Füllungsdrücke als Ausdruck einer sich entwickelnden Herzinsuffizienz während eines Spontanatemversuchs können echokardiographisch erfasst werden [33]. Ein Anstieg des zentralen Venendrucks während eines Spontanatemversuchs ist möglicherweise ebenfalls Ausdruck einer sich entwickelnden Herzinsuffizienz. Wenn ein Spontanatemversuch wegen eines kardiogenen Lungenödems scheitert, lässt sich anhand des Anstiegs des Gesamtproteins im Plasma eine Hämokonzentration nachweisen. Ein Anstieg des Gesamtproteins im Plasma $>9 \%$ am Ende des gescheiterten Spontanatemversuches im Vergleich zu unmittelbar vor dem Spontanatemversuch ist hochspezifisch für ein weaninginduziertes kardiogenes Lungenödem, während ein Anstieg < $3 \%$ das Problem mit hoher Wahrscheinlichkeit ausschließt [34].

Die therapeutische Strategie bei weaninginduzierter Herzinsuffizienz umfasst positiv inotrope Medikation, sowie eine
Reduktion der Vorlast mittels Entwässerung. Es ist auch empfehlenswert, diese Patienten unmittelbar nach einer gelungenen Extubation nichtinvasiv mittels CPAP weiter zu unterstützen und so die myokardiale Arbeitslast zu reduzieren. Durch eine Dauerinfusion von Nitroglycerin $(40-600 \mu \mathrm{g} / \mathrm{min})$ kann neben der Vorlastsenkung auch die bei Spontanatemversuchen regelhaft auftretende systemische arterielle Hypertonie verhindert und somit die Nachlast vermindert werden [35].

Im Rahmen des unter Spontanatmung gesteigerten myokardialen $\mathrm{O}_{2}$-Verbrauches kann eine prä-existente koronare Herzkrankheit mit einem akuten Koronarsyndrom wirksam werden [36]. Gelegentlich können diese Patienten erst nach einer koronaren Revaskularisation erfolgreich entwöhnt werden.

\section{Neuromuskuläre Limitationen}

Eine erfolgreiche Entwöhnung von der Beatmung erfordert einen intakten Atemantrieb mit gesicherter neuraler Übertragung in die Atemmuskulatur, die ausreichende Kraft und Ausdauer besitzen muss. Störungen auf jeder Ebene dieser Kette können zu prolongierter Entwöhnung führen.
Zentrale Atemdepression. Abgesehen von primären zerebralen Pathologien wie Blutungen und Insulte sind inadäquat dosierte Sedativa eine häufige Ursache von Atemdepression. Auch die metabolische Alkalose mit Alkalämie vermindert den Atemantrieb [37]. Der Atemantrieb kann anhand des $\mathrm{P}_{\mathrm{o}, 1}$ (Atemwegsokklusionsdruck innerhalb von $100 \mathrm{~ms}$ ) abgeschätzt werden. Ein $\mathrm{P}_{\mathrm{o}, 1}<1$ mbar spricht für einen reduzierten Atemantrieb, ein $\mathrm{P}_{\mathrm{o}, 1}>6 \mathrm{mbar}$ entspricht dagegen einem pathologisch gesteigerten Atemantrieb wie bei drohender Erschöpfung der Atempumpe [38]. Die Therapie einer derartigen Störung sollte möglichst kausal sein. Eine metabolische Alkalose mit Alkalämie sollte kausal und ggf. mit einer einmaligen Gabe von $500 \mathrm{mg}$ Azetazolamid behandelt werden[39]. Ansonsten haben Medikamente, die den Atemantrieb direkt steigern, keine Bedeutung in der Entwöhnung.

\section{Muskuläre Schwäche bei kritischer} Krankheit. Während und nach kritischer Krankheit kommt es regelhaft zu ausgeprägter Schwäche der quergestreiften Muskulatur („ICU-aquired weakness“). Neben der Inaktivitätshypotrophie in Folge von Langzeitsedierung und kontrollierter Beatmung spielen spezifische neuromuskuläre Abnormitäten bei kritischer 
Krankheit (CINMA, „critical-illness neuromuscular abnormalities") eine Rolle.

CINMA beschreibt eine Gruppe peripherer neuromuskulärer Dysfunktionen, die nach kritischer Krankheit auftreten und üblicherweise sowohl Nerven und Muskeln betreffen. Bei komplexer Ätiopathogenese sind die bekannten Risikofaktoren

- Sepsis,

- Mehrorganversagen,

- Kortikosteroidtherapie und

- Hyperglykämie [40].

Typischerweise ist die proximale Extremitätenmuskulatur bilateral betroffen, die Sensorik ist oft unbeeinträchtigt. CINMA betrifft oft auch die Atemmuskulatur, was den Zusammenhang zwischen CINMA mit prolongierter Respiratorentwöhnung und Bedarf an Tracheotomie erklärt [41, 42].

Die Diagnose von CINMA ist schwierig: Die Wahrscheinlichkeit kann klinisch anhand von Scores abgeschätzt werden, eine definitive Diagnose erfordert eine neurophysiologische Abklärung und ggf. histologische Sicherung. Die neuromuskulären Funktionen kehren in der Regel nach Wochen bis Monaten wieder zurück, eine wirksame Therapie gibt es bislang nicht.

Die Dekonditionierung (Trainingsmangel) des Intensivpatienten kann mittels physikalischer Trainingstherapie behandelt werden. Dadurch verbessern sich Muskelkraft und funktioneller Status und die Beatmungsdauer wird verkürzt [43].

Beatmungsassoziierte Zwerchfelldysfunktion. Experimentelle Daten legen nahe, dass kontrollierte Beatmung die Kraft des Zwerchfells reduziert. Der Mechanismus dürfte am ehesten eine Inaktivitätshypotrophie sein, wenngleich auch oxydativer Stress und andere Mechanismen eine Rolle spielen [44].

Ob eine beatmungsassoziierte Zwerchfelldysfunktion (VIDD, ,ventilator-induced diaphragm dysfunction") eine prolongierte Respiratorentwöhnung verursachen kann, ist unklar [45]. Eine sichere Diagnose der VIDD und auch die Abgrenzung zu CINMA sind in der Praxis oft schwierig. Präventive Ansätze sind assistierte Atemmodi mit permissiver spon- taner Zwerchfellaktivität, N.-phrenicusStimulation und Antioxidanzien [44].

\section{Neuropsychiatrische Limitationen}

Delir. Das Delir ist Ausdruck einer akuten und reversiblen zerebralen Dysfunktion und eine häufige Komplikation bei kritischer Krankheit. Es verlängert die Beatmungsdauer und ist mit erhöhter Sterblichkeit verbunden $[46,47,48]$. Die Diagnose gelingt mithilfe einer standardisierten klinischen Untersuchung [49]. Neben den wichtigen nichtpharmakologischen Maßnahmen werden v. a. Neuroleptika eingesetzt.

Angst, Depression und Schlafstörungen. Dies sind sehr häufige Probleme von Patienten mit prolongierter Entwöhnung. Das Vorhandensein von depressiven Symptomen konnte als unabhängiger Risikofaktor für das Versterben von Patienten mit prolongierter Entwöhnung ermittelt werden [50]. Wenngleich ein direkter Zusammenhang mit dem Erfolg der Entwöhnung bislang nicht gezeigt wurde, können Panikattacken während der Spontanatemphasen im Einzelfall ein systematisches Training der Atempumpe und der peripheren Muskulatur unmöglich machen. Entscheidend ist die Vermeidung dieser Probleme u. a. durch ein oberflächliches Sedierungsregime [51] sowie schonende pharmakologische und nichtpharmakologische anxiolytische und antidepressive Therapien. Mit Biofeedbacktechniken kann Angst erfolgreich behandelt und die Entwöhnungsdauer verkürzt werden [52].

\section{Metabolische und endokrine Störungen}

Wenngleich klare Daten fehlen, ist es nahe liegend, dass schwere Elektrolytstörungen die Entwöhnbarkeit behindern können. Dies trifft v. a. auf Hypo- und Hypernatriämie und die damit verbundenen zerebralen Dysfunktionen zu. Störungen des Kalium-, Magnesium- und Phosphathaushalts wirken sich ungünstig auf neuromuskuläre Funktionen aus und sollten im Rahmen von prolongierter Entwöhnung behoben werden. Eine manifeste Hypothyreose oder ein Hypo- aldosteronismus können die Entwöhnung ebenfalls verhindern.

\section{Ernährung}

Adipositas führt nicht zu einer erschwerten Entwöhnung vom Respirator [53]. Ob Katabolismus und Malnutrition explizite Risikofaktoren für prolongierte Entwöhnung darstellen, ist nicht bekannt. Es erscheint jedoch sinnvoll und wird von Daten aus nichtkontrollierten Studien nahegelegt, während der äußerst energieaufwändigen Phase der Entwöhnung eine entsprechend hohe Kalorienzufuhr sicherzustellen [54].

\section{Anämie}

Bei kritisch kranken COPD-Patienten führt die Korrektur einer Anämie zu einer Reduktion der Atemarbeit [55]. Ob eine liberale Transfusionspolitik mit Aufrechterhalten von $\mathrm{Hb}$-Werten $>10 \mathrm{~g} / \mathrm{dl}$ eine prolongierte Entwöhnung entscheidend verkürzen kann, ist nicht klar. Zumindest konnte die Gesamtbeatmungsdauer eines allgemein kritisch kranken Kollektivs durch eine derartige Intervention nicht verkürzt werden [56].

\section{Prävention}

Entscheidende Strategien zur Vermeidung prolongierter Entwöhnung sind - der zurückhaltende Umgang mit Langzeitsedierung,

- die Durchführung von Sedierungspausen und

- rechtzeitige Spontanatemversuche.

Durch derartige Interventionen können die Beatmungsdauer verkürzt und die Mortalität gesenkt werden $[20,57]$. Das Zulassen von residualer Spontanatemaktivität kann möglicherweise der Inaktivitätshypotrophie des Zwerchfells während kontrollierter Beatmung vorbeugen.

Risikopatienten für lange Beatmungsdauer und prolongierte Entwöhnung früh zu identifizieren, ist im Einzelfall nicht verlässlich möglich. Generell gilt jedoch, dass eine schwere akute Erkrankung vor dem Hintergrund einer vorbestehenden Lungenkrankheit bei Vorliegen von Muskelschwäche mit hoher Wahrscheinlichkeit zu prolongierter Entwöhnung führen wird [58]. 


\section{Patientenmanagement}

Reversible Ursachen für prolongierte Entwöhnung müssen erfasst und nach Möglichkeit behandelt werden. Entscheidend ist hier, bereits früh anhand einer systematischen Diagnostik spezifische Probleme auszuschließen bzw. nachzuweisen (• Tab. 3).

Die frühe und aggressive Rehabilitation auf der Intensivstation ist vermutlich die einzige Intervention, die der Schwäche der Extremitätenmuskulatur entgegenwirken kann. Das Training der Atemmuskulatur kann mittels intermittierender Spontanatemphasen oder mittels Atmung gegen einen dosierten Flusswiderstand durchgeführt werden.

\section{- Durch eine Tracheotomie werden Kommunikation, Nahrungsaufnahme und Bronchialtoilette üblicherweise positiv beeinflusst.}

In der Praxis werden daher viele Patienten mit prolongierter Beatmung über eine Tracheotomie entwöhnt. Wenngleich eine frühe Tracheotomie zu einer kürzeren Beatmungsdauer führt, wird die Mortalität nicht positiv beeinflusst [59]. Da im Einzelfall die Notwendigkeit und der Vorteil einer Tracheotomie nicht verlässlich prognostiziert werden kann, kann die routinemäßige Frühtracheotomie nicht empfohlen werden.

Spezialisierte Entwöhnungseinrichtungen können im Verbund mit einem Akutspital („step-down unit“) oder als Entwöhnungszentrum („single standing weaning center") eingerichtet werden. Der Transfer von Patienten mit prolongierter Entwöhnung in eine derartige Einrichtung sollte nach Möglichkeit früh erfolgen, nicht zuletzt um die Intensivbettenkapazitäten zu schonen und die Kosten zu reduzieren $[60,61]$. Telemedizinisch gestützte Überwachung kann in Zukunft möglicherweise die Entwöhnung stabiler Patienten in anderen Versorgungseinrichtungen oder sogar zuhause ermöglichen [62].

Vor dem Hintergrund ihrer eingeschränkten Prognose und der teilweise beträchtlich reduzierten Lebensqualität ist es bei Patienten mit prolongierter Entwöhnung entscheidend, von Beginn an neben der angestrebten Entwöhnung auch eine palliative Strategie zu verfolgen. Die aktive Auseinandersetzung mit den ethischen Herausforderungen, die sich im Zuge der Behandlung dieser schwerkranken Patienten ergeben, kann die Qualität von Entscheidungen über Therapiebegrenzung und Therapiebeendigung positiv beeinflussen $[63,64]$.

\section{Fazit für die Praxis}

- Da potenziell entwöhnbare Patienten oft übersehen werden, sollte die Entwöhnung schon früh in Betracht gezogen und nach einem klaren Schema strukturiert durchgeführt werden.

- „Ready-to-wean“-Kriterien sind u. a.: Ursache für die Beatmung gebessert, stabile Hämodynamik, stabiler Gasaustausch unter geringem Beatmungsaufwand, kräftiger Hustenstoß.

- Ein kurzer Spontanatemversuch (SBT) dient als Screeningtest auf Entwöhnbarkeit. Bei einem $\mathrm{RSBl}<100$ nach 2 min wird der SBT verlängert: Ist der Patient nach 30-120 min respiratorisch, hämodynamisch und zerebral stabil, kann und soll extubiert werden.

- Patienten mit prolongierter Entwöhnung haben eine deutlich erhöhte Mortalität. Wenn ein SBT negativ ausfällt, sollte daher gezielt nach den dafür verantwortlichen Faktoren gesucht werden.

- Ist die Entwöhnung aus respiratorischen Gründen limitiert, sollte die Atemarbeit minimiert und eine schwache Atemmuskulatur trainiert werden.

- Die Senkung von Vor- und Nachlast sowie ggf. positiv-inotrope Therapie und ggf. koronare Revaskularisierung sind Optionen, wenn die Entwöhnung aus kardialen Gründen scheitert.

- Zur Vermeidung von Weaningproblemen sollte sparsam mit Sedierung umgegangen werden. Muskulär schwache Patienten sollten möglichst früh rehabilitiert werden.

\section{Korrespondenzadresse PD Dr. G.-C. Funk

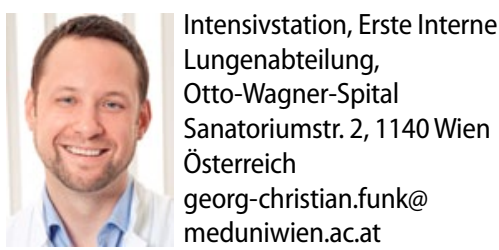

Interessenkonflikt. Der korrespondierende Autor gibt an, dass kein Interessenkonflikt besteht.

\section{Literatur}

\section{Das Literaturverzeichnis ...}

... finden Sie in der html-Version dieses Beitrags im Online-Archiv auf der Zeitschriftenhomepage www.intensivmedizin.springer.de 
Hier steht eine Anzeige.

黛 Springer 